\title{
Protective effect of dexmedetomidine against organ dysfunction in a two-hit model of hemorrhage/ resuscitation and endotoxemia in rats
}

\author{
Yuanxu Jiang (iD ${ }^{1}$, Mingzhu Xia ${ }^{2}$, Qiang Huang ${ }^{1}$, Dengfeng Ding ${ }^{1}$, Yali $\mathrm{Li}^{1}$, \\ Zhongjun Zhang ${ }^{1}$ and Xueping Zhang (i) ${ }^{1}$ \\ ${ }^{1}$ Department of Anesthesiology, Shenzhen People's Hospital, Shenzhen Anesthesiology Engineering Center, \\ the Second Clinical Medical College, Jinan University, Shenzhen, China \\ ${ }^{2}$ Hubei Community Health Service Center, Luohu Hospital group, Shenzhen, China
}

\begin{abstract}
Dexmedetomidine (DEX), a selective agonist of $\alpha_{2}$-adrenergic receptors, has anti-inflammation properties and potential beneficial effects against trauma, shock, or infection. Therefore, this study aimed to investigate whether DEX might protect against multiple-organ dysfunction in a two-hit model of hemorrhage/resuscitation (HS) and subsequent endotoxemia. Eighty Wistar rats were randomized into four groups: NS (normal saline), HS/L (HS plus lipopolysaccharide), HS/L + D (HS/L plus dexmedetomidine), and $\mathrm{HS} / \mathrm{L}+\mathrm{D}+\mathrm{Y}\left(\mathrm{HS} / \mathrm{L}+\mathrm{D}\right.$ plus yohimbine). Six hours after resuscitation, blood gas $\left(\mathrm{PaO}_{2}\right)$ and serum alanine aminotransferase (ALT), aspartate aminotransferase (AST), blood urine nitrogen (BUN), creatinine (Cr), TNF- $\alpha$, IL- $\beta$, IL-6, IL-8, IL-10, and nitric oxide (NO) were measured. The histopathology was assayed by staining. Malondialdehyde (MDA) and superoxide dismutase (SOD) levels and heme oxygenase-1 $(\mathrm{HO}-1)$ were assayed. The $\mathrm{PaO}_{2}$ levels in $\mathrm{HS} / \mathrm{L}$ rats were lower whereas the ALT, AST, BUN, Cr, TNF- $\alpha$, IL- $\beta$, IL-6, IL-8, IL-10, and NO levels were higher compared to the control group. The $\mathrm{HS} / \mathrm{L}+\mathrm{D}$ increased $\mathrm{PaO}_{2}$ and further increased IL-10 and decreased ALT, AST, BUN, Cr, TNF- $\alpha$, IL- $\beta$, IL-6, IL-8, and NO levels of the $\mathrm{HS} / \mathrm{L}$ groups. In addition, the MDA in the HS/L groups increased whereas SOD activity decreased compared to the control group. Moreover, the HO-1 expression levels were increased by DEX administration in lung, liver, and kidney tissues. Lungs, livers, and kidneys of the HS/L group displayed significant damage, but such damage was attenuated in the HS/L + D group. All of the above-mentioned effects of DEX were partly reversed by yohimbine. DEX reduced multiple organ injury caused by $\mathrm{HS} / \mathrm{L}$ in rats, which may be mediated, at least in part, by $\alpha_{2}$-adrenergic receptors.
\end{abstract}

Key words: Hemorrhagic shock; Resuscitation; Endotoxemia; Dexmedetomidine; Yohimbine

\section{Introduction}

The current two-hit hypothesis suggests that trauma (hemorrhage/resuscitation) creates a systemic inflammatory environment that is more sensitive to bacterial toxins (1). Endotoxemia induces the production of various inflammatory cytokines such as tumor necrosis factor (TNF- $\alpha$ ) and interleukin-1ß (IL-1 $\beta)$ as well as reactive oxygen species (ROS). These mediators alone, or through their interactions, lead to neutrophil accumulation, local inflammation, and secretion of secondary proinflammatory cytokines and chemokines that eventually trigger a systemic inflammatory response syndrome and multiple organ dysfunction (MOD) (2).

Hemorrhagic shock leads to impaired delivery of oxygen to organs, and the main management during the first hit is to maintain oxygen delivery to prevent hypoxia, ischemia, inflammation, and organ dysfunction. This is achieved with the use of fluids, vasopressors, and blood transfusion (3). If endotoxemia occurs, the standard approach also includes fluids and vasopressors in addition to respiratory and renal support (4). However, these approaches are ineffective at controlling the inflammatory response.

Dexmedetomidine (DEX), a selective agonist of $\alpha_{2}$ adrenergic receptors, has sedative, analgesic, and sympatholytic properties, and it has been used for analgesia, sedation, and anesthetic sparing. Apart from its sedative and analgesic properties, DEX has also demonstrated potential anti-inflammatory effects both in vivo and in vitro $(5,6)$. In addition, DEX has been shown to protect organs against ischemia-reperfusion $(\mathrm{I} / \mathrm{R})$ injury and sepsis in experimental animal models (7-9).

However, it remains unknown whether DEX could exert multiple organ protective effects in a model of hemorrhage/

Correspondence: Xueping Zhang: <13613051840@163.com> 
resuscitation and subsequent endotoxemia. Therefore, this study aimed to examine the effects of DEX on lung injury, renal injury, and hepatic injury in a two-hit rat model of hemorrhage/resuscitation and endotoxemia.

\section{Material and Methods}

\section{Animals \\ Eighty male Wistar rats (200-220 g, 6-8 weeks old) raised in a specific pathogen-free environment were provided by the Experimental Animal Center of Nanfang Medical University (China). The rats were housed in an environmentally controlled animal care facility where they were fed ad libitum and exposed to 12-h light/dark cycles. The protocol was approved by the Animal Care Committee of Nanfang Medical University and was carried out according to institutional guidelines for animal care and by the Guide for Care and Use of Laboratory Animals published by the United States National Institutes of Health.}

\section{Two-hit model protocol}

The two-hit model was induced as previously described (10). A PE-50 catheter was inserted into the left carotid artery to monitor blood pressure and into the right carotid artery to induce hemorrhage. Control animals underwent catheter insertion, but no blood was withdrawn or returned. Hemorrhage was induced by withdrawing blood into a heparinized syringe $(0.025 \mathrm{~mL} / \mathrm{g}$ weight) for $10 \mathrm{~min}$ to lower mean arterial pressure (MAP) to approximately $40-50 \mathrm{mmHg}$. MAP was constantly maintained by withdrawing or infusing blood, as needed, during a 60 min period. Then, resuscitation was performed by reinfusing the remaining withdrawn blood plus normal saline (2-fold the maximum blood volume drawn) over $10 \mathrm{~min}$. Sixty minutes after resuscitation, Escherichia coli endotoxin lipopolysaccharide (LPS), $15 \mathrm{mg} / \mathrm{kg}$, Serotype 0127:B8; Sigma-Aldrich, USA) was administered intravenously to induce endotoxemia.

\section{Animal preparation and experimental design}

After having their skin shaved and disinfected with $70 \%$ ethanol, the rats were anesthetized with sodium pentobarbital (50 mg/kg, ip injection). The right carotid artery and left femoral vein of each rat were punctured with catheters (PE-50) for administration of drugs and measurements of hemodynamic parameters. A tracheostomy was performed, and a 14-gauge angiocatheter was inserted as a tracheostomy tube to keep the airway unobstructed. After tracheostomy, anesthesia was maintained by supplementary injections of pentobarbital (approximately $1-3 \mathrm{mg} / \mathrm{kg}$ per hour, iv), as required.

The animals were randomly assigned to four groups ( $n=20$ /group): normal saline control group (NS), hemorrhage/resuscitation plus LPS group (HS/L), HS/L + DEX group $(H S / L+D)$, and $H S / L+D E X+$ yohimbine group $(H S / L+D+Y)$. Rats in the NS group were treated with $0.9 \%$ normal saline $(5 \mathrm{~mL} / \mathrm{kg})$, without causing hemorrhage or endotoxemia. The HS/L + D group received DEX $(1 \mu \mathrm{g} /$ $\mathrm{kg}$, intravenous infusion over $10 \mathrm{~min}$ ), followed by a second DEX infusion ( $5.0 \mu \mathrm{g} / \mathrm{kg}$ per hour) beginning immediately after endotoxin administration and lasting until the end of the experiment $(6 \mathrm{~h})$. The DEX dose was determined based on previous studies (11). Rats in the $H S / L+D+Y$ group received yohimbine $(0.1 \mathrm{mg} / \mathrm{kg}$, intravenous infusion over $5 \mathrm{~min}$ ) immediately after the loading dose of DEX.

The arterial catheter was connected to a pressure transducer (P23ID, Statham, USA) for taking measurements of MAP and heart rate (HR), which were continuously recorded on a multichannel recorder (MacLab/4e, AD Instruments Pty Ltd., Australia). At the end of the experiment, $1.5 \mathrm{~mL}$ of blood was collected from each animal into a serum gel S/1.3 tube (Monovette, Sarstedt, Germany) from a catheter placed in the carotid artery and used to measure liver and renal function. Another sample of blood $(1.0 \mathrm{~mL})$ was collected for blood gas analysis. All rats were sacrificed by intravenous injection with high-dose pentobarbital $(300 \mathrm{mg} / \mathrm{kg}$ ). Samples of lung, liver, and kidney tissues were removed for further examination.

The study consisted of two parts. The first part was the survival study, for which 10 animals were used in each group. The second part was the biochemical and histological study, for which another 10 animals were used in each group. If animals died, new animals were modeled to maintain the number of rats at 10 for each group/experiment. Therefore, for the first part, 10 rats from each group were randomly selected and used to determine the survival rate at $72 \mathrm{~h}$ after LPS administration.

\section{Arterial oxygen tension $\left(\mathrm{PaO}_{2}\right)$}

A $0.5-\mathrm{mL}$ blood sample was collected from the right common carotid artery of each rat $6 \mathrm{~h}$ after LPS administration, and $\mathrm{PaO}_{2}$ was immediately measured with a blood gas analyzer (Stat Profile pHOx, Nova Biomedical Corporation, USA).

\section{Biochemical indicators of organ injury}

A 2-mL blood sample was collected from the right common carotid artery of each rat $6 \mathrm{~h}$ after LPS administration, blood samples were immediately centrifuged ( $2500 \mathrm{~g}$ for $10 \mathrm{~min}$ at $4^{\circ} \mathrm{C}$ ) and the serum was used to measure the levels of aspartate aminotransferase (AST), alanine aminotransferase (ALT), blood urine nitrogen (BUN), and creatinine (Cr) (Fuji DRI-CHEM 3030, Fuji Photo Film, Japan). The remaining serum was immediately stored at $-20^{\circ} \mathrm{C}$ for subsequent measurements.

\section{Malondialdehyde (MDA) and superoxide dismutase (SOD) activity in lung, liver, and kidney tissues}

The lung, liver, and kidney tissues were removed $6 \mathrm{~h}$ after LPS administration, snap-frozen in liquid nitrogen and then stored at $-80^{\circ} \mathrm{C}$ for subsequent analysis. The tissues were homogenized and centrifuged (2500 $\mathrm{g}$ for $10 \mathrm{~min}$ at $4^{\circ} \mathrm{C}$ ) and the supernatant was incubated in a 
water bath $\left(60^{\circ} \mathrm{C}\right)$ for $2 \mathrm{~h}$ for subsequent determination of MDA levels and SOD activity. The assay kits for SOD and MDA were purchased from Jianchen Bioengineering Institute (China).

\section{Plasma cytokines and NO concentration}

A 2-mL blood sample was collected from the right common carotid artery of each rat $6 \mathrm{~h}$ after LPS administration. After $30 \mathrm{~min}$, the blood samples were centrifuged (2500 $\mathrm{g}$ for $10 \mathrm{~min}$ at $4^{\circ} \mathrm{C}$ ) and the serum samples were stored at $-20^{\circ} \mathrm{C}$ for subsequent analysis. The serum levels of TNF- $\alpha$, IL-1 $1 \beta$, IL-6, IL-8, and IL-10 were analyzed using enzyme-linked immunosorbent assay kits (R\&D Systems, USA), according to the manufacturer's instructions. The nitric oxide (NO) concentration in tissue was determined by nitrate reductase according to the manufacturer's instructions.

\section{Histological examination}

Samples of lung, kidney, and liver tissues were taken $6 \mathrm{~h}$ after LPS administration. The specimens were routinely fixed and embedded in paraffin. Sections were stained with hematoxylin and eosin (H\&E). A semiquantitative scoring system was adopted to evaluate the lung, liver, and kidney injury in a blind manner using light microscopy.

The histological lung injury was scored based on the alveolar congestion, hemorrhage, neutrophil infiltration into the airspace or vessel wall, and thickness of the alveolar wall (12). The histological liver injury was scored based on hepatocellular necrosis, bleeding, and inflammatory cell infiltration in the liver (12). The histological kidney injury was scored based on the inflammatory cell infiltration and glomerular capillary congestion (12). Lung, kidney, and liver sections were scored as 1: no pathological changes or very slight; 2: slight pathological changes; 3: moderate pathological changes; 4: severe pathological changes. Evaluation scores were added for the total lung, kidney, and liver renal injury score.

\section{Western blot analysis}

The appropriate amount of lung, liver, and kidney tissue (50 mg) was taken separately, the protein lysis liquid was added, the samples were homogenized on ice, and centrifuged at $12,000 \mathrm{~g}$ for $20 \mathrm{~min}$ at $4^{\circ} \mathrm{C}$, and supernatants collected. The protein concentration was determined by a bicinchoninic acid protein assay. Samples with equal quantities of protein were mixed with $5 \times$ SDS sample buffer and then boiled for $15 \mathrm{~min}$. Aliquots of the samples were separated by $10 \%$ SDS-PAGE and electrophoresis. Following transfer to polyvinylidene fluoride (PVDF) membranes, the samples were blocked with TBS $+20 \%$ Tween 20 (TBST) solution containing 5\% skimmed milk powder for $1.5 \mathrm{~h}$ at room temperature. The membranes were washed 3 times with TBST at room temperature, 10 min each time, and then incubated overnight at $4^{\circ} \mathrm{C}$ with $\mathrm{HO}-1$ primary antibody (1:1000 dilution, Abcam, USA) and an anti- $\beta$-actin antibody (1:1000 dilution, Santa Cruz Biotechnology, USA). The membranes were washed 3 times with TBST at room temperature, 10 min each time, and then incubated with an HRP-conjugated secondary antibody for $2 \mathrm{~h}$ at room temperature. Membranes were washed 3 times with TBST at room temperature, $10 \mathrm{~min}$ each time, then reacted with an enhanced chemiluminescence substrate (Pierce, USA), and finally exposed to photographic film for a suitable length of time. The images were analyzed using ImageJ software ( $\mathrm{NIH}, \mathrm{USA})$, and the ratios of $\mathrm{HO}-1$ provided a measurement of the HO-1 levels.

\section{Statistical analysis}

Data are reported as means $\pm S D$. The significance of differences among the four groups was tested using analysis of variance (ANOVA) for normal distribution data. Multiple comparisons were subjected to Dunnett's test. Survival analysis was done using the Kaplan-Meier method, and comparisons between groups were made using the logrank test. All statistical analyses were performed using SPSS 11.0 for Windows (SPSS Inc., USA). Two-sided $\mathrm{P}$-values $<0.05$ were considered statistically significant.

\section{Results}

\section{Effects of DEX on the hemodynamics of rats with hemorrhage/resuscitation and endotoxemia}

There were no significant differences among the groups regarding animal weight, blood loss, resuscitation volume, and the dose of pentobarbital (all $\mathrm{P}>0.05$ ) (Table 1).

Table 1. Baseline characteristics.

\begin{tabular}{lcccc}
\hline Group & Weight $(\mathrm{g})$ & Blood loss volume $(\mathrm{mL})$ & Resuscitation volume $(\mathrm{mL})$ & Dose of pentobarbital $(\mathrm{mg})$ \\
\hline NS & $208.40 \pm 4.09$ & 0 & 0 & $14.17 \pm 0.28$ \\
HS/L & $207.60 \pm 4.01$ & $5.19 \pm 0.10$ & $10.26 \pm 0.10$ & $14.12 \pm 0.27$ \\
HS/L + D & $209.10 \pm 5.13$ & $5.23 \pm 0.13$ & $10.41 \pm 0.25$ & $14.22 \pm 0.35$ \\
HS/L + D +Y & $205.30 \pm 4.06$ & $5.18 \pm 0.11$ & $10.25 \pm 0.18$ & $13.98 \pm 0.26$ \\
\hline
\end{tabular}

Data are reported as means $\pm S D(n=10)$. There were no significant differences among the groups regarding animal weight, blood loss, resuscitation volume, and the dose of pentobarbital (all $\mathrm{P}>0.05$, ANOVA). NS: normal saline; HS/L: hemorrhage/resuscitation plus lipopolysaccharide; D: dexmedetomidine; Y: yohimbine. 


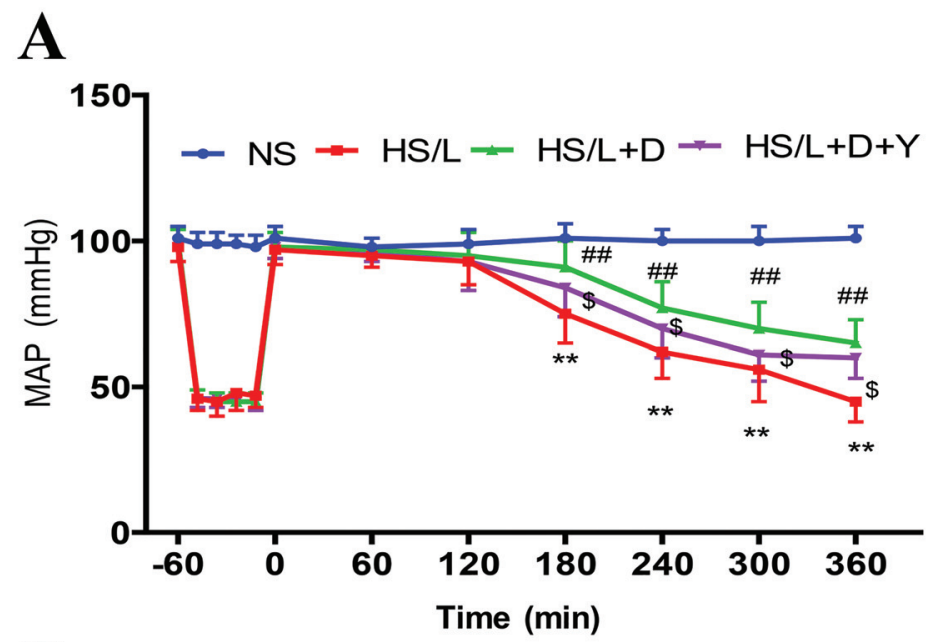

Figure 1. Changes in mean arterial pressure (MAP) (A) and heart rate (HR) (B) from the NS control (normal saline), hemorrhage/resuscitation plus lipopolysaccharide (HS/L), HS/L plus dexmedetomidine $(H S / L+D)$, and $H S / L+D$ plus yohimbine $(H S / L+D+Y)$ groups. Data are reported as means $\pm S D$. ${ }^{*} P<0.01,{ }^{*} P<0.05$ vs the NS group; ${ }^{\# \#} \mathrm{P}<0.01,{ }^{\#} \mathrm{P}<0.05$ vs the $\mathrm{HS} / \mathrm{L}$ group; ${ }^{\$} \mathrm{P}<0.05$ vs the $\mathrm{HS} / \mathrm{L}+\mathrm{D}$ group (ANOVA). $n=10 /$ group.

B

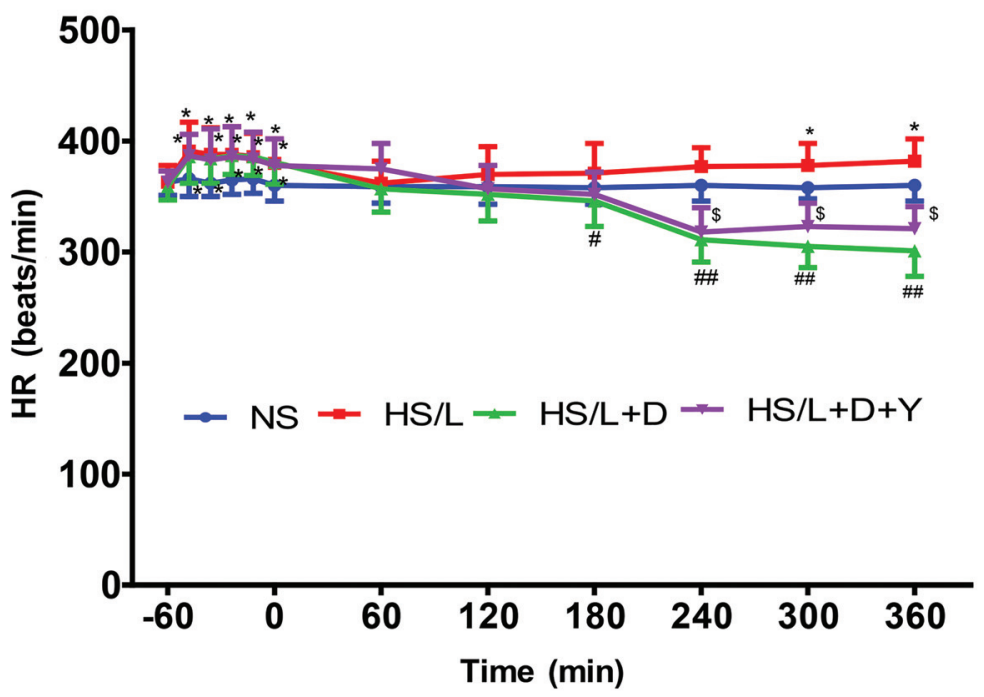

Values of MAP in the NS group remained stable throughout the experiment (Figure 1A and B). Baseline values for MAP in the NS, HS/L, HS/L+D, and $H S / L+D+Y$ groups were similar (all $\mathrm{P}>0.05$ ), and values for MAP 1 to $2 \mathrm{~h}$ after resuscitation in the $N S, H S / L, H S / L+D$, and $H S / L+D+Y$ groups were also similar (all $P>0.05$ ). However, values for MAP measured from 3 to $6 \mathrm{~h}$ after resuscitation in the HS/L, $H S / L+D$, and $H S / L+D+Y$ groups were significantly lower than those in the $N / S$ group (all $P<0.01$ ). Values for MAP in the $H S / L+D$ group were significantly higher than those in the $\mathrm{HS} / \mathrm{L}$ group from 3 to $6 \mathrm{~h}$ after resuscitation (all $P<0.01$ ), and MAP values in the $H S / L+D+Y$ group were higher than those in the $H S / L+D$ group (all $P<0.05$ ) (Figure 1A).

Baseline values for $\mathrm{HR}$ in the NS, HS/L, HS/L + D, and $\mathrm{HS} / \mathrm{L}+\mathrm{D}+\mathrm{Y}$ groups were similar (all $\mathrm{P}>0.05$ ). $\mathrm{HR}$ was measured every $12 \mathrm{~min}$ from -60 to $0 \mathrm{~min}$ and every $1 \mathrm{~h}$ after resuscitation. $\mathrm{HR}$ values measured from -60 to $0 \mathrm{~min}$ in the $H S / L, H S / L+D$, and $H S / L+D+Y$ groups were higher than those in the NS group $(P<0.05)$. HR values measured from 5 to $6 \mathrm{~h}$ in the HS/L group were significantly higher than those in the NS group $(P<0.05)$. Values for HR in the HS/L + D group were significantly lower than those in the $\mathrm{HS} / \mathrm{L}$ group from 3 to $6 \mathrm{~h}$ after resuscitation $(P<0.05$, $P<0.01)$. Values for $H R$ in the $H S / L+D+Y$ group were higher than those in the HS/L $+D$ group from 4 to $6 \mathrm{~h}$ after resuscitation (all $\mathrm{P}<0.05$ ) (Figure $1 \mathrm{~B}$ ).

\section{Effects of DEX on organ dysfunction caused by} hemorrhage/resuscitation plus endotoxemia in rats

Biochemical assays revealed that HS plus LPS administration resulted in increased serum levels of ALT, AST, BUN, and $\mathrm{Cr}$ (all $\mathrm{P}<0.01$ ) (Figure 2A to 2D). However, treatment of HS/L rats with DEX attenuated all 
A

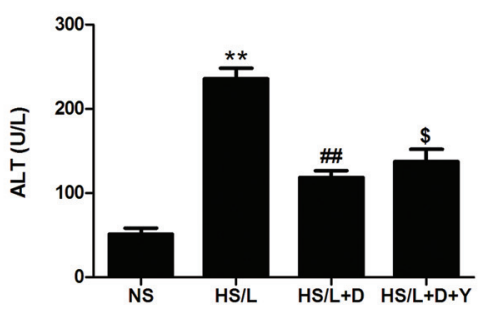

D

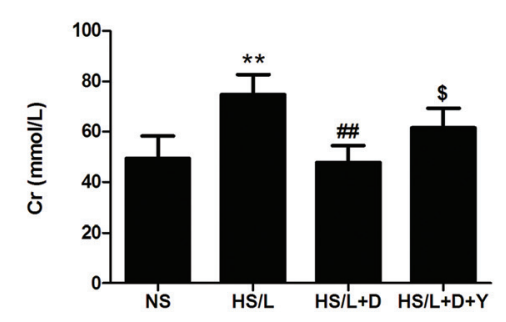

B

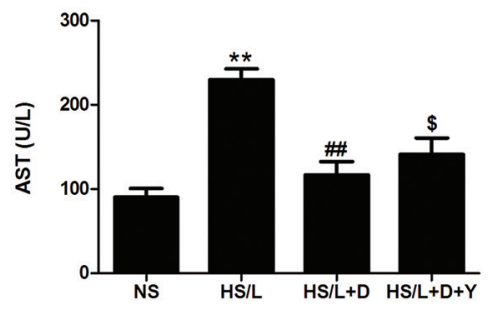

C

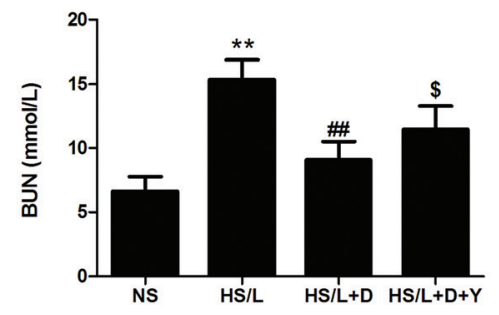

E

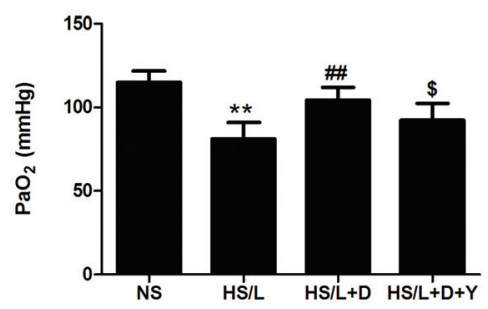

Figure 2. Plasma levels of (A) alanine aminotransferase (ALT), (B) aspartate aminotransferase (AST), (C) blood urine nitrogen (BUN), (D) creatinine ( $\mathrm{Cr})$, and $(\mathrm{E})$ blood gas $\left(\mathrm{PaO}_{2}\right)$ in the $\mathrm{NS}$ control (normal saline), hemorrhage/resuscitation plus lipopolysaccharide (HS/L), HS/L plus dexmedetomidine (HS/L +D), and HS/L + D plus yohimbine $(H S / L+D+Y)$ groups. Data are reported as means $\pm \mathrm{SD}$. ${ }^{* *} \mathrm{P}<0.01$ vs the NS group; ${ }^{\# \#} \mathrm{P}<0.01$ vs the HS/L group; ${ }^{\$} \mathrm{P}<0.05$ vs the HS/L + D group (ANOVA). $n=10 /$ group.

of these increases. In addition, the levels of ALT, AST, $B U N$, and $\mathrm{Cr}$ in the $\mathrm{HS} / \mathrm{L}+\mathrm{D}+\mathrm{Y}$ group were higher than those in the $H S / L+D$ group (all $P<0.05$ ). Arterial blood gas data indicated that rats in the $\mathrm{HS} / \mathrm{L}$ group had decreased $\mathrm{PaO}_{2}$ (lung function) levels $(\mathrm{P}<0.01)$, which were increased by DEX administration, but $\mathrm{PaO}_{2}$ levels in the $H S / L+D+Y$ group were lower than those in the $H S / L+D$ group $(P<0.05)$.

\section{Effects of DEX on MDA levels and SOD activity induced by hemorrhage/resuscitation plus endotoxemia in rats}

Compared with the NS group, rats in the HS/L group had significantly increased MDA levels and reduced SOD activity in lung, liver, and kidney tissues (all $P<0.01$ ), while rats in the HS/L+D group showed significantly decreased MDA levels and increased SOD activity in those tissues compared with the HS/L group (all $P<0.01$ ). The increases in MDA levels and reductions in SOD activity in lung, liver, and kidney tissue were partially reversed by administration of yohimbine (all $\mathrm{P}<0.05$ ) (Figure $3 \mathrm{~A}$ and $\mathrm{B}$ ).

Effects of DEX on plasma levels of NO and the proinflammatory and anti-inflammatory cytokines induced by hemorrhage/resuscitation plus endotoxemia in rats

Compared with the NS group, rats in the HS/L group had significantly increased plasma levels of TNF- $\alpha, \mathrm{IL}-\beta$,
IL-6, IL-8, and NO (all $\mathrm{P}<0.01$, Figure 4A-E). Compared with the $H S / L$ group, rats in the $H S / L+D$ group had significantly decreased plasma levels of TNF- $\alpha$, IL- $\beta$, IL- 6 , $\mathrm{IL}-8$, and NO (all $\mathrm{P}<0.01$ ), while these increases were partially reversed in the $H S / L+D+Y$ group $(P<0.05)$. Compared with the NS group, rats in the HS/L group had significantly increased plasma levels of $I L-10(P<0.01)$. Compared with the HS/L group, rats in the HS/L + D group had further increased plasma levels of IL-10 (Figure 4F) $(P<0.01)$, while these increases were partially reversed in the $\mathrm{HS} / \mathrm{L}+\mathrm{D}+\mathrm{Y}$ group $(\mathrm{P}<0.05)$.

Effects of DEX on the histopathology of lung, liver, and kidney caused by hemorrhage/resuscitation plus endotoxemia in rats

Histological analyses revealed normal lung, liver, and kidney tissues in the NS group (Figure 5A, E, I); severe lung, liver, and kidney injuries in the HS/L group (Figure 5B, F, J); and only mild-to-moderate lung, liver, and kidney injuries in the $H S / L+D$ (Figure $5 C, G$, $\mathrm{K}$ ) and $\mathrm{HS} / \mathrm{L}+\mathrm{D}+\mathrm{Y}$ groups (Figure $5 \mathrm{D}, \mathrm{H}, \mathrm{L}$ ). Compared with the NS group, rats in the HS/L group showed higher lung, liver, and kidney injury scores (all $\mathrm{P}<0.01$ ). Compared with the HS/L group, rats in the $\mathrm{HS} / \mathrm{L}+$ $D$ group showed lower lung, liver, and kidney (Figure 5 $\mathrm{M}-\mathrm{O}$ ) injury scores (all $\mathrm{P}<0.01$ ), while these increases were partially reversed in the $H S / L+D+Y$ group $(\mathrm{P}<0.05)$. 


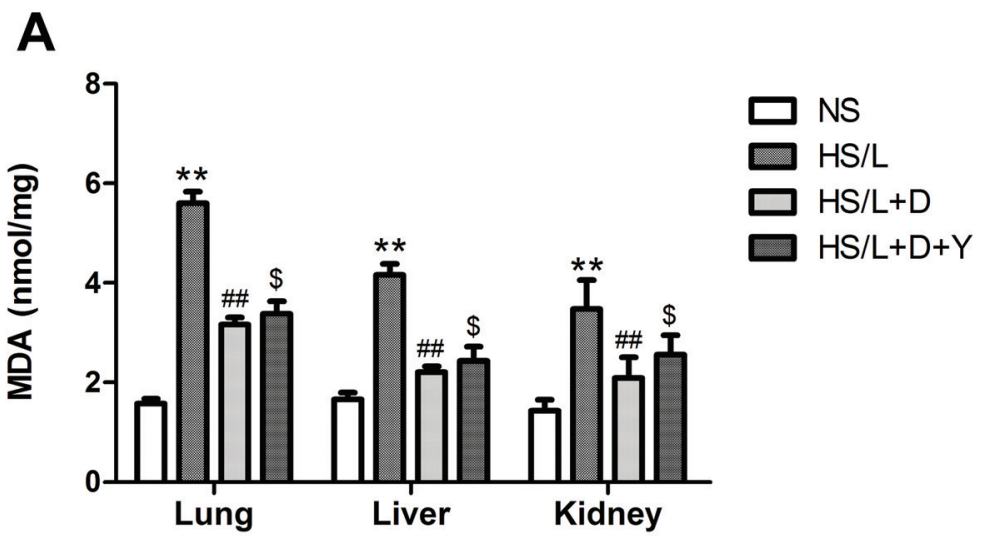

Figure 3. Malondialdehyde (MDA) levels (A) and superoxide dismutase (SOD) activity (B) in lung, liver, and kidney tissues of the NS control (normal saline), hemorrhage/resuscitation plus lipopolysaccharide (HS/L), HS/L plus dexmedetomidine $(H S / L+D)$, and $H S / L+D$ plus yohimbine $(H S / L+$ $D+Y)$ groups. Data are reported as means $\pm S D$. ${ }^{* *} \mathrm{P}<0.01$ vs the NS group; ${ }^{\# \#} \mathrm{P}<0.01$ vs the HS/ L group; ${ }^{\$} \mathrm{P}<0.05$ vs the HS/L+D group (ANOVA). n=10/group.

B

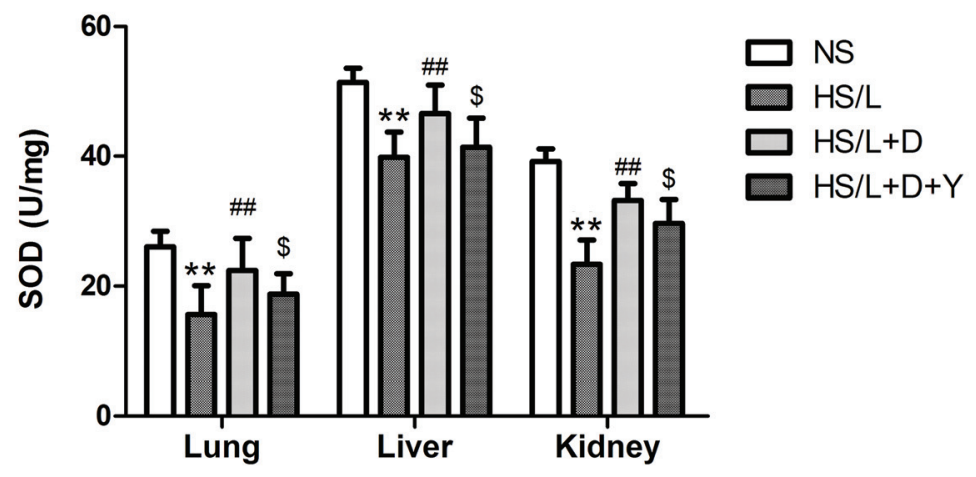

A

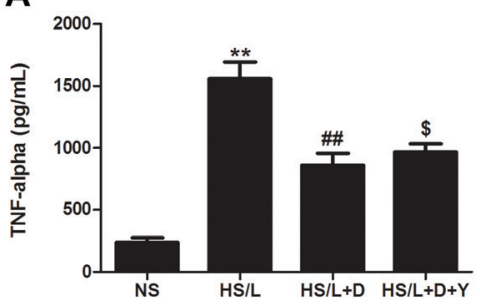

D

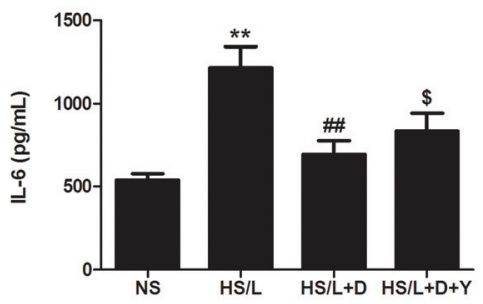

B

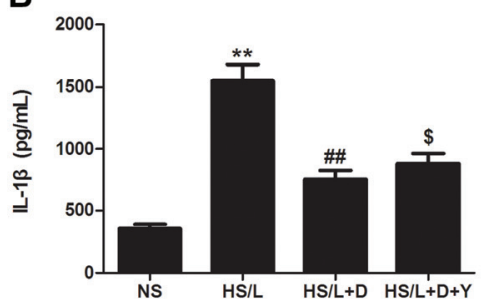

E

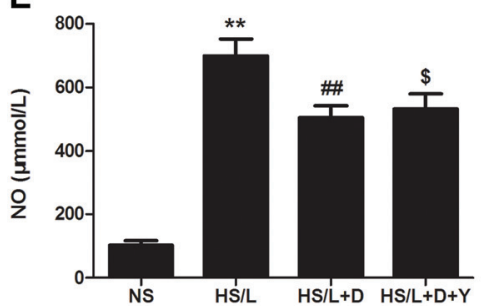

C

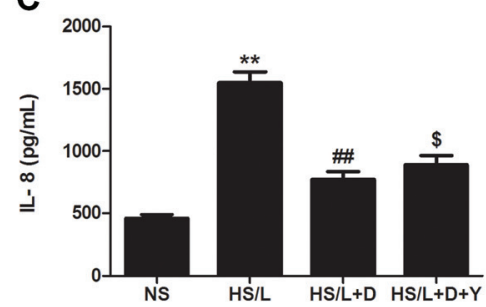

$\mathbf{F}$

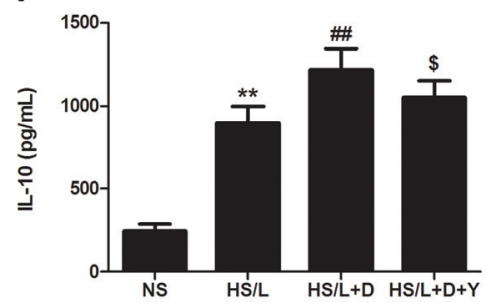

Figure 4. Plasma levels of TNF- $\alpha$ (A), IL-1 $\beta$ (B), IL-8 (C), IL-6 (D), NO (E) and IL-10 (F) of the NS control (normal saline), hemorrhage/ resuscitation plus lipopolysaccharide $(H S / L), H S / L$ plus dexmedetomidine $(H S / L+D)$, and $H S / L+D$ plus yohimbine $(H S / L+D+Y)$ groups. Data are reported as means $\pm \mathrm{SD}$. ${ }^{*} \mathrm{P}<0.01$ vs the NS group; ${ }^{\#} \mathrm{P}<0.01$ vs the HS/L group; ${ }^{\$} \mathrm{P}<0.05$ vs the HS/L $+\mathrm{D}$ group (ANOVA). $n=10 /$ group. 

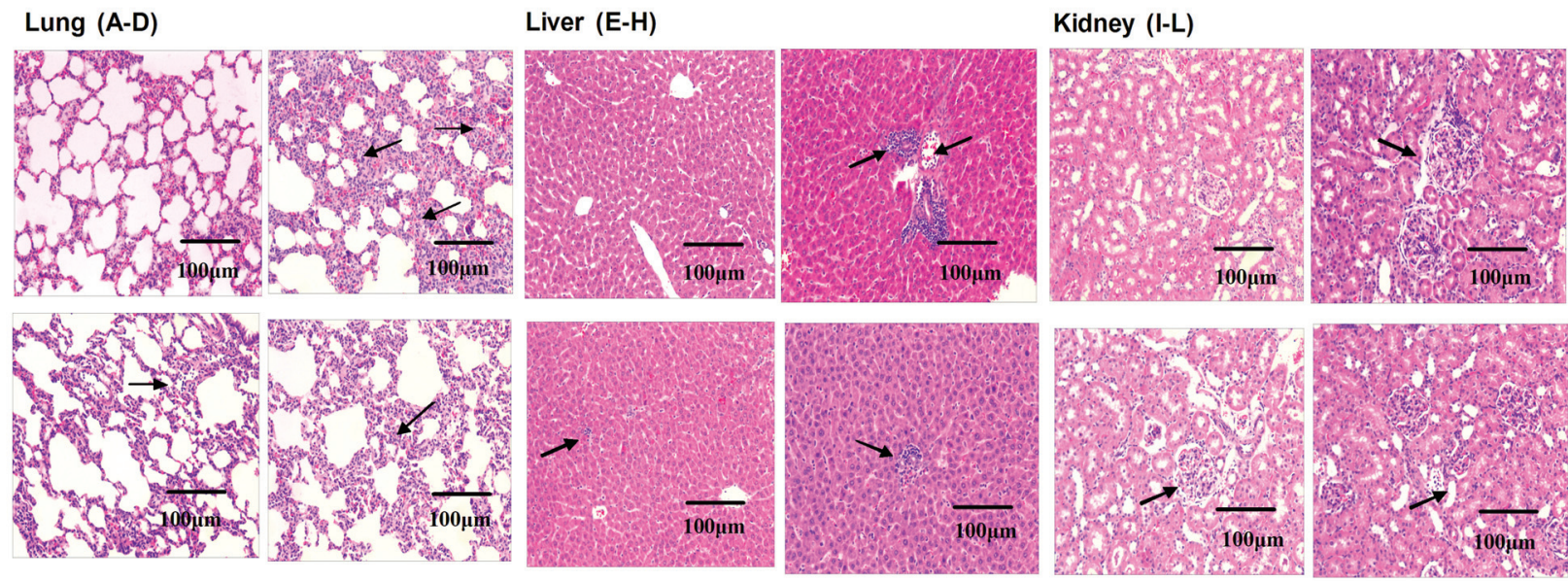

Lung (M)

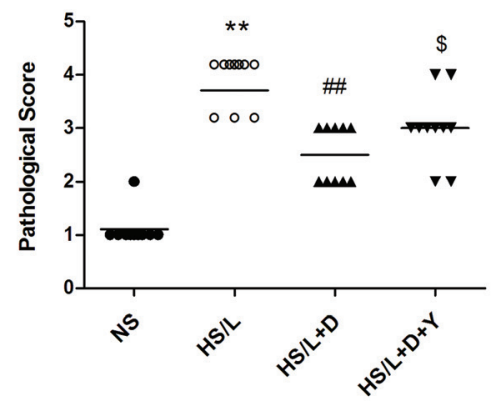

Liver (N)

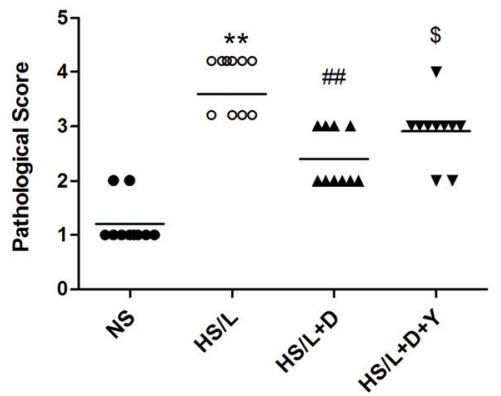

Kidney (0)

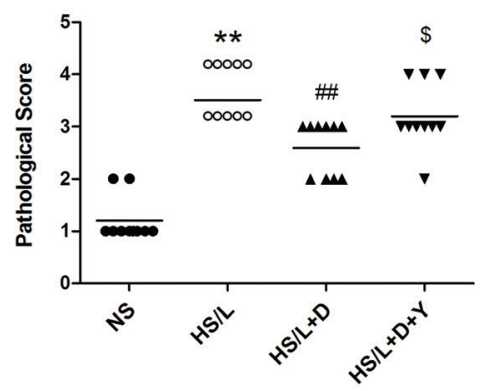

Figure 5. Representative microscopic findings of the lung (A-D), liver (E-H), and kidney (I-L) tissues of the NS control (normal saline, $n=10)$, hemorrhage/resuscitation plus lipopolysaccharide $(H S / L, n=10), H S / L$ plus dexmedetomidine $(H S / L+D$, $n=10)$, and $H S / L+D$ plus yohimbine $(H S / L+D+Y, n=10)$ groups (hematoxylin and eosin; $\times 200$, magnification bar: $100 \mu m$ ). Arrows indicate pathological changes. Histological injury scores for lung (M), liver $(\mathbf{N})$, and kidney (O) tissues. Data are reported as means $\pm \mathrm{SD}$. ${ }^{* *} \mathrm{P}<0.01$ vs the NS group; ${ }^{\# \#} \mathrm{P}<0.01$ vs the HS/L group; ${ }^{\$} \mathrm{P}<0.05$ vs the HS/L + D group (ANOVA). $\mathrm{n}=10 /$ group.

\section{Effects of DEX on HO-1 protein expression of lung, liver, and kidney caused by hemorrhage/resuscitation plus endotoxemia in rats \\ Compared with the NS group, rats in the HS/L group had increased HO-1 protein levels in lung, liver, and kidney tissue $(P<0.01)$. Compared with the HS/L group, rats in the $\mathrm{HS} / \mathrm{L}+\mathrm{D}$ group had significantly increased $\mathrm{HO}-1$ protein levels in lung, liver, and kidney tissue (Figure 6A-C) $(P<0.01)$, while these increases were partially reversed in the $H S / L+D+Y$ group $(P<0.05)$.}

Effects of DEX on the survival rate of rats treated with hemorrhage/resuscitation plus endotoxemia

No rats died in the NS group, but all rats in the HS/L group died within $54 \mathrm{~h}$ after LPS injection. Compared with the $H S / L$ group, rats in the $H S / L+D$ group had an increased survival rate within $72 \mathrm{~h}(\mathrm{P}<0.01)$, while these increases were partially reversed in the $H S / L+D+Y$ group $(P<0.05)$ (Figure 7 ).

\section{Discussion}

Previous studies were mainly limited to the protective effect of dexmedetomidine on a single organ induced by a model (7-9), but the clinical reality is more complicated. Hemorrhage/resuscitation and subsequent endotoxemia are common in clinical practice, and multiple organ damage induced by this two-hit model is more in line with clinical practice. Therefore, we chose a hemorrhage/resuscitation and subsequent endotoxemia two-hit model to observe whether dexmedetomidine had a protective effect on multiple organs such as the lung, liver, and kidney. Our study showed for the first time that DEX can attenuate lung injuries, as well as renal and liver dysfunction, in a two-hit model of hemorrhage/ resuscitation and endotoxemia in rats. Our results also showed that DEX improved survival in rats with HS/L. In addition, the multiorgan protective effects of DEX were partly reversed by yohimbine, an antagonist of the 
A
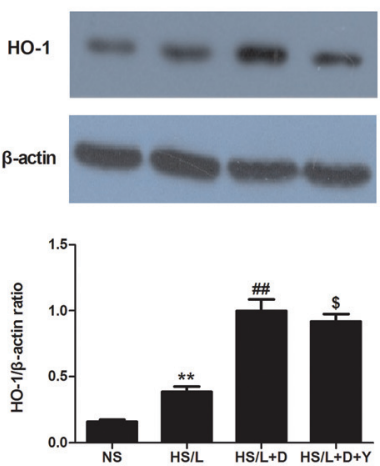
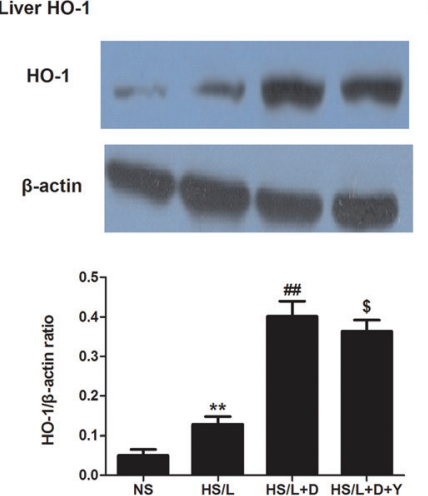
Kidney HO-1
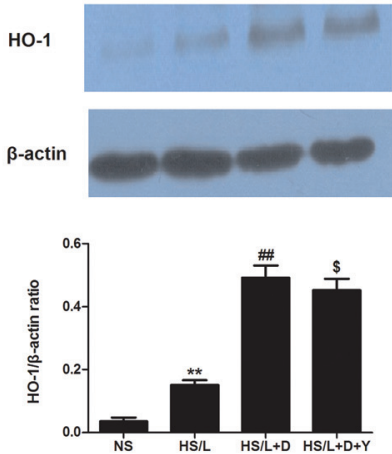

Figure 6. Heme oxygenase-1 (HO-1) protein expression in the lung (A), liver (B), and kidney (C) of the NS control (normal saline), hemorrhage/resuscitation plus lipopolysaccharide (HS/L), HS/L plus dexmedetomidine (HS/L + D), and HS/L+D plus yohimbine (HS/ $\mathrm{L}+\mathrm{D}+\mathrm{Y})$ groups. Data are reported as means $\pm \mathrm{SD}$. ${ }^{*} \mathrm{P}<0.01$ vs the NS group; ${ }^{\# \#} \mathrm{P}<0.01$ vs the HS/L group; ${ }^{\$} \mathrm{P}<0.05$ vs the HS/L + D group (ANOVA). $n=10 /$ group.

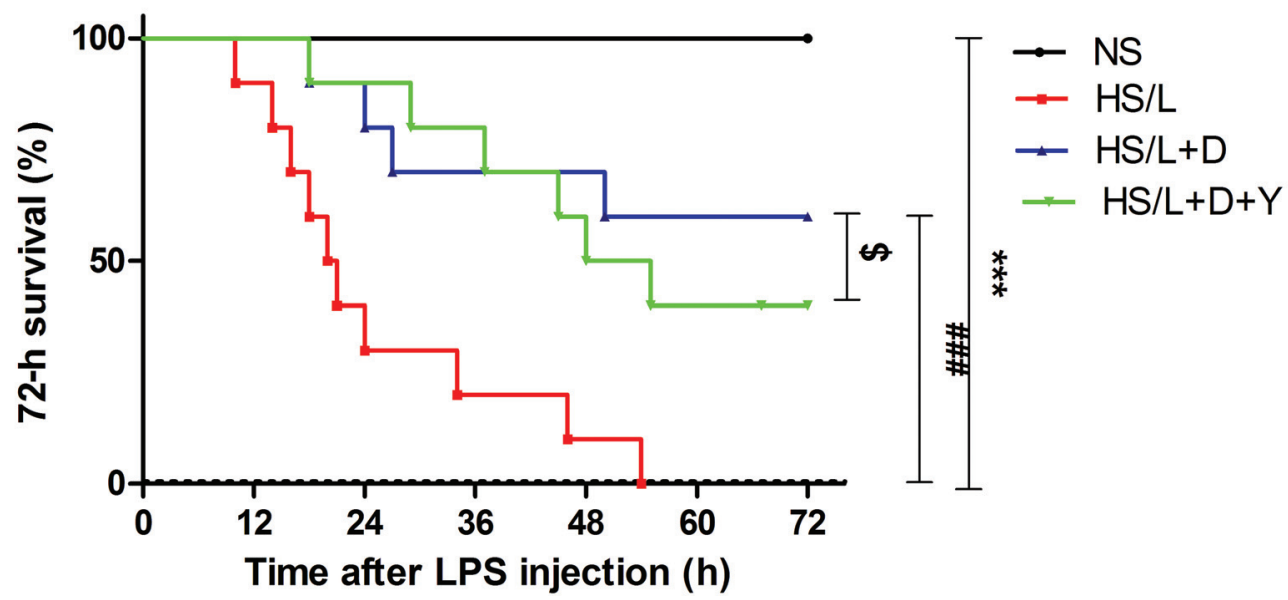

Figure 7. Comparison of 72-h survival of rats treated with normal saline (NS), hemorrhage/resuscitation plus lipopolysaccharide (HS/L), $\mathrm{HS} / \mathrm{L}$ plus dexmedetomidine $(H S / L+D)$, and $H S / L+D$ plus yohimbine $(H S / L+D+Y)$. ${ }^{* *} P<0.001$ vs $N S$ group; ${ }^{\# \#} P<0.001$ vs HS/ $L$ group; ${ }^{\$} P<0.05$ vs HS/L + D group (ANOVA). $n=10 /$ group.

$\alpha_{2}$-adrenergic receptors. DEX has been reported to provide effective sedative effects to critically ill patients (13). The findings of the present study could be beneficial to patients with hemorrhage/resuscitation and subsequent endotoxemia.

The data also revealed that DEX reduced inflammation resulting from damaged lung, liver, and kidney tissue, and serum released after hemorrhage/resuscitation and subsequent endotoxemia, thereby increasing the survival of HS/L-treated rats. These data seem to support the concept that DEX might be beneficial in clinical situations similar to those represented in the HS/L group of rats. However, previous studies have administered DEX at doses higher than a clinical dose to show that it effectively attenuated an inflammatory response or organ injury (14).
Actual clinical trials are required to verify the ability of DEX to protect organs in this clinical context.

The increased release of proinflammatory cytokines plays an important role in the initiation and perpetuation of organ injury (15). Remick et al. (16) reported that IL-6 levels $6 \mathrm{~h}$ after induction of sepsis were predictive of mortality in a rodent model of sepsis. Furthermore, Kraft et al. (17) demonstrated that elevated levels of IL-8 correlated with increased rates of MOD, sepsis, and mortality. Liu et al. (18) showed that increased levels of TNF- $\alpha$ and TNF receptors (TNFRs) were associated with the severity of trauma-provoked organ dysfunction. These combined data suggest that serum levels of proinflammatory cytokines may be a valid biomarker for monitoring organ dysfunction. Previous studies revealed 
that DEX decreased LPS-induced levels of TNF- $\alpha$, IL-1, IL-6, and IL-8 production in both murine macrophages (19) and human whole blood in vitro (20). When proinflammatory cytokines are released, anti-inflammatory cytokines are also released. In a previous study, IL-10 inhibited the release of TNF- $\alpha$ in mice induced by LPS and reduced the mortality of a lethal dose of LPS (21). Wu et al. reported that IL-10 therapy could inhibit the activation of neutrophils and the secretion of proinflammatory cytokines, which is protective in LPS-induced ALI (22). In the present study, increased plasma levels of TNF- $\alpha$, IL- $\beta$, IL-6, and IL- 8 induced by HS/L could be attenuated by DEX treatment. The results also showed that hemorrhage/ resuscitation induced an increase in IL-10 levels and that DEX treatment further increased IL-10 levels, indicating that the protective properties of DEX against organ dysfunction are associated with reduced proinflammatory and increased anti-inflammatory processes.

Oxidative stress has been shown to play an important role in organ failure (23). SOD exerts strong antioxidant effects. MDA is the end-product of lipid peroxidation. In this study, the levels of MDA and SOD were assessed in lung, liver, and kidney tissues to evaluate the effects of DEX on oxidative stress. The results suggested that organ injuries in the HS/L rats resulted in increased MDA levels and decreased SOD activity, which could be reversed by DEX. DEX exerts potent antioxidant effects (24). Furthermore, DEX has been shown to decrease MDA levels and increase SOD activity in patients with lung cancer receiving one-lung ventilation (25). These findings suggest that the mechanisms underlying the therapeutic effects of DEX in humans may be similar to those that allow it to attenuate oxidative stress in HS/L-treated rats.

The hemodynamic data of the present study indicated that $H S / L$ decreased MAP and increased HR in rats, while administration of DEX increased MAP and reduced HR. Thus, it is likely that the mechanism by which DEX alleviates hemodynamic alterations may also contribute to its therapeutic effects. A previous study reported that DEX increased vascular reactivity, reduced vasopressor requirements in cases of septic shock, and increased blood pressure (26). Another study reported that DEX increased the pressor response to norepinephrine without adverse consequences on tissue perfusion in rats with sepsis. $(27,28)$ Although the alfa-2 adrenergic receptor is a known mechanism of controlling blood pressure by negative feedback on sympathetic neuronal fibers, this inhibitory effect of dexmedetomidine has a small effect on blood pressure $(11,29)$. In addition, inflammatory cytokines such as TNF- $\alpha$, IL- $\beta$, and NO can inhibit the systolic function of the heart and dilating peripheral blood vessels (30-32), and these inflammatory cytokines can damage the capillary endothelial cells, causing capillary leakage, thereby reducing the effective circulation capacity (33). Therefore, the above factors are the main causes of hypotension induced by hemorrhage/resuscitation and subsequent endotoxemia. In this study, we observed that DEX inhibited the release of these inflammatory cytokines induced by hemorrhage/resuscitation and subsequent endotoxemia. In summary, DEX prevented a further blood pressure drop mainly by inhibiting the release of inflammatory cytokines, while the influence of alfa-2 adrenergic receptor-mediated sympathetic negative feedback was relatively small.

$\mathrm{HO}-1$, also known as heat shock protein 32 , is a ratelimiting enzyme that catalyzes the production of heme. Studies have shown that $\mathrm{HO}-1$ plays anti-inflammatory and antioxidant roles in a mouse model of acute pancreatitis

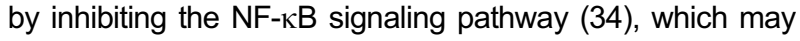
be beneficial for increasing blood pressure. Although the $\mathrm{HO}-1 /$ carbon monoxide pathway is a known vascular diastolic mechanism, carbon monoxide at the same time has been shown to play anti-inflammatory, antioxidant, and organ-protective roles $(35,36)$. As a result, $\mathrm{HO}-1$ exerts anti-inflammatory effects not only by inhibiting the $\mathrm{NF}-\kappa \mathrm{B}$ signaling pathway but also through the production of the anti-inflammatory compound carbon monoxide. Enhanced HO-1 expression after hemorrhagic shock and subsequent resuscitation in multiple organs (including the kidney, liver, and lung) might be responsible for the organ protection and inflammation inhibitory effects (37). Recently, Gao et al. (25) reported that DEX increased the levels of $\mathrm{HO}-1$ expression and reduced oxidative stress during one-lung ventilation. In the present study, DEX treatment resulted in significantly higher $\mathrm{HO}-1$ protein expression in major organs, including the lung, liver, and kidney. Therefore, DEX-induced increased MAP may be mainly related to the anti-inflammatory and antioxidant effects of $\mathrm{HO}-1$, and the effect of $\mathrm{HO}-1 / \mathrm{CO}$ pathway vasodilatation on blood pressure is relatively small.

$\alpha_{2}$-adrenoceptors were first discovered in the presynapse of the adrenergic neurons in the central and peripheral nervous systems. The physiologic responses elicited by activation of $\alpha_{2}$-adrenergic receptors include decreased salivation and pain and increased sedation. Nevertheless, a recent study by Takahashi et al. (38) has shown that DEX inhibited glucose-stimulated insulin secretion in a concentration-dependent manner, but this effect was reversed by yohimbine, an $\alpha 2$-adrenoceptor blocker. Another study also reported that yohimbine could completely neutralize the protective effect of DEX on oxidative damage of alveolar macrophages induced by $\mathrm{H}_{2} \mathrm{O}_{2}$ (39). These studies suggest that $\alpha 2$-adrenoceptors may also be present in some non-neuronal cell types in humans. It was previously reported that activation of $\alpha_{2}$-adrenergic receptors decreased the levels of various inflammatory factors and protected against LPS-induced acute kidney injuries (8). Shen et al. (7) reported that stimulation of the $\alpha_{2}$-adrenergic receptors reduced pulmonary damage and inhibited sterile inflammation induced by lung $\mathrm{I} / \mathrm{R}$ injuries. In the present study, administration of an 
$\alpha_{2}$-adrenergic receptor antagonist (yohimbine) reduced the effects of DEX on the inflammatory response and oxidant stress and counteracted the organ protective effects of DEX. These results suggest that DEX protected against HS/L-induced multiorgan injury by activating $\alpha_{2}$-adrenergic receptors. Clinically, DEX is usually used to calm patients in critical situations. Our data and previous studies showed that DEX reduces the inflammatory response and organ damage induced by hemorrhagic shock and infection. Future research should focus on the anti-inflammatory effect of $\alpha 2$-adrenergic receptor and whether DEX is effective at treating critically ill patients.

\section{References}

1. Moore FA, Moore EE. Evolving concepts in the pathogenesis of postinjury multiple organ failure. Surg Clin North Am 1995; 75: 257-277, doi: 10.1016/S0039-6109(16)46587-4.

2. Ramirez M. Multiple organ dysfunction syndrome. Curr Probl Pediatr Adolesc Health Care 2013; 43: 273-277, doi: 10.1016/j.cppeds.2013.10.003.

3. Bougle A, Harrois A, Duranteau J. Resuscitative strategies in traumatic hemorrhagic shock. Ann Intensive Care 2013; 3: 1, doi: 10.1186/2110-5820-3-1.

4. Russell JA, Walley KR. Update in sepsis 2012. Am J Respir Crit Care Med 2013; 187: 1303-1307, doi: 10.1164/rccm. 201303-0567UP.

5. Peng M, Wang YL, Wang CY, Chen $\mathrm{C}$. Dexmedetomidine attenuates lipopolysaccharide-induced proinflammatory response in primary microglia. J Surg Res 2013; 179: e219-e225, doi: 10.1016/j.jss.2012.05.047.

6. Xu L, Bao H, Si Y, Wang X. Effects of dexmedetomidine on early and late cytokines during polymicrobial sepsis in mice. Inflamm Res 2013; 62: 507-514, doi: 10.1007/s00011-0130604-5.

7. Shen J, Fu G, Jiang L, Xu J, Li L, Fu G. Effect of dexmedetomidine pretreatment on lung injury following intestinal ischemia-reperfusion. Exp Ther Med 2013; 6: 1359-1364, doi: 10.3892/etm.2013.1317.

8. Tan F, Chen Y, Yuan D, Gong C, Li X, Zhou S. Dexmedetomidine protects against acute kidney injury through downregulating inflammatory reactions in endotoxemia rats. Biomed Rep 2015; 3: 365-370, doi: 10.3892/br.2015.427.

9. Ibacache M, Sanchez G, Pedrozo Z, Galvez F, Humeres C, Echevarria G, et al. Dexmedetomidine preconditioning activates pro-survival kinases and attenuates regional ischemia/ reperfusion injury in rat heart. Biochim Biophys Acta 2012; 1822: 537-545, doi: 10.1016/j.bbadis.2011.12.013.

10. Chen KB, Lee CY, Lee JJ, Tsai PS, Huang CJ. Platonin mitigates lung injury in a two-hit model of hemorrhage/ resuscitation and endotoxemia in rats. J Trauma Acute Care Surg 2012; 72: 660-670, doi: 10.1097/TA.0b013e31 82318551.

11. Taniguchi $\mathrm{T}$, Kidani $\mathrm{Y}$, Kanakura $\mathrm{H}$, Takemoto $\mathrm{Y}$, Yamamoto $\mathrm{K}$. Effects of dexmedetomidine on mortality rate and inflammatory responses to endotoxin-induced shock in rats. Crit Care Med 2004; 32: 1322-1326, doi: 10.1097/01.CCM. 0000128579.84228.2A.
In summary, DEX mitigated multiple-organ injury in a two-hit rat model of hemorrhagic/resuscitation and subsequent endotoxemia by inhibiting both the inflammatory response and oxidative stress through the induction of $\mathrm{HO}-1$. These effects were mediated, at least in part, by $\alpha$;2-adrenoceptors.

\section{Acknowledgments}

The authors acknowledge the help of Dr. Jing-Xu with the pathological examination of the tissues. This study was funded by the Technical Research and Development Fund of Shenzhen (JCYJ20160422142317026).

12. Zhang XP, Zhang L, Wang $Y$, Cheng QH, Wang JM, Cai W, et al. Study of the protective effects of dexamethasone on multiple organ injury in rats with severe acute pancreatitis. JOP 2007; 8: 400-412.

13. Constantin JM, Momon A, Mantz J, Payen JF, De Jonghe B, Perbet $S$, et al. Efficacy and safety of sedation with dexmedetomidine in critical care patients: a meta-analysis of randomized controlled trials. Anaesth Crit Care Pain Med 2016; 35: 7-15, doi: 10.1016/j.accpm.2015.06.012.

14. Shi $Q Q$, Wang $H$, Fang $H$. Dose-response and mechanism of protective functions of selective alpha-2 agonist dexmedetomidine on acute lung injury in rats. Saudi Med J 2012; 33: 375-381.

15. Hatakeyama N, Matsuda N. Alert cell strategy: mechanisms of inflammatory response and organ protection. Curr Pharm Des 2014; 20: 5766-5778, doi: 10.2174/138161282036140 912122809.

16. Remick DG, Bolgos G, Copeland S, Siddiqui J. Role of interleukin-6 in mortality from and physiologic response to sepsis. Infect Immun 2005; 73: 2751-2757, doi: 10.1128/ IAI.73.5.2751-2757.2005.

17. Kraft R, Herndon DN, Finnerty CC, Cox RA, Song J, Jeschke MG. Predictive value of IL-8 for sepsis and severe infections after burn injury: a clinical study. Shock 2015; 43: 222-227, doi: 10.1097/SHK.0000000000000294.

18. Liu C, Tang J. Expression levels of tumor necrosis factoralpha and the corresponding receptors are correlated with trauma severity. Oncol Lett 2014; 8: 2747-2751, doi: 10.3892/ ol.2014.2575.

19. Lai YC, Tsai PS, Huang CJ. Effects of dexmedetomidine on regulating endotoxin-induced up-regulation of inflammatory molecules in murine macrophages. J Surg Res 2009; 154: 212-219, doi: 10.1016/j.jss.2008.07.010.

20. Kawasaki T, Kawasaki C, Ueki M, Hamada K, Habe K, Sata T. Dexmedetomidine suppresses proinflammatory mediator production in human whole blood in vitro. $J$ Trauma Acute Care Surg 2013; 74: 1370-1375, doi: 10.1097/TA.0b013e3 $1828 \mathrm{db} 978$.

21. Gerard C, Bruyns C, Marchant A, Abramowicz D, Vandenabeele $P$, Delvaux $A$, et al. Interleukin 10 reduces the release of tumor necrosis factor and prevents lethality in experimental endotoxemia. J Exp Med 1993; 177: 547-550, doi: 10.1084/jem.177.2.547. 
22. Wu CL, Lin LY, Yang JS, Chan MC, Hsueh CM. Attenuation of lipopolysaccharide-induced acute lung injury by treatment with IL-10. Respirology 2009; 14: 511-521, doi: 10.1111/ j.1440-1843.2009.01516.x.

23. Abraham E, Singer M. Mechanisms of sepsis-induced organ dysfunction. Crit Care Med 2007; 35: 2408-2416, doi: 10.1097/01.CCM.0000282072.56245.91.

24. Erer D, Ozer A, Arslan M, Oktar GL, Iriz E, Elmas C, et al. The protective effects of dexmedetomidine on liver injuryinduced myocardial ischemia reperfusion. Bratisl Lek Listy 2014; 115: 422-426.

25. Gao S, Wang Y, Zhao J, Su A. Effects of dexmedetomidine pretreatment on heme oxygenase-1 expression and oxidative stress during one-lung ventilation. Int $J$ Clin Exp Pathol 2015; 8: 3144-3149.

26. Pichot C, Geloen A, Ghignone M, Quintin L. Alpha-2 agonists to reduce vasopressor requirements in septic shock? Med Hypotheses 2010; 75: 652-656, doi: 10.1016/j.mehy.2010. 08.010.

27. Geloen A, Chapelier K, Cividjian A, Dantony E, Rabilloud M, May $\mathrm{CN}$, et al. Clonidine and dexmedetomidine increase the pressor response to norepinephrine in experimental sepsis: a pilot study. Crit Care Med 2013; 41: e431-e438, doi: 10.1097/CCM.0b013e3182986248.

28. Miranda ML, Balarini MM, Bouskela E. Dexmedetomidine attenuates the microcirculatory derangements evoked by experimental sepsis. Anesthesiology 2015; 122: 619-630, doi: 10.1097/ALN.0000000000000491.

29. Hernández G, Tapia P, Alegría L, Soto D, Luengo C, Gomez $\mathrm{J}$, et al. Effects of dexmedetomidine and esmolol on systemic hemodynamics and exogenous lactate clearance in early experimental septic. Crit Care 2016; 20: 234, doi: 10.1186/ s13054-016-1419-x.

30. Flierl MA, Rittirsch D, Huber-Lang MS, Sarma JV, Ward PA. Molecular events in the cardiomyopathy of sepsis. Mol Med 2008; 4: 327-336.

31. Fallach $R$, Shainberg $A$, Avlas $O$, Fainblut $M$, Chepurko $Y$, Porat E, et al. Cardiomyocyte Toll-like receptor 4 is involved in heart dysfunction following septic shock or myocardial ischemia. J Mol Cell Cardiol 2010; 48: 1236-1244, doi: 10.1016/j.yjmcc.2010.02.020.

32. Hochstadt A, Meroz Y, Landesberg G. Myocardial dysfunction in severe sepsis and septic shock: more questions than answers? J Cardiothorac Vasc Anesth 2011; 25: 526-535, doi: 10.1053/j.jvca.2010.11.026.

33. Aird WC. The role of the endothelium in severe sepsis and multiple organ dysfunction syndrome. Blood 2003; 101: 3765-3777, doi: 10.1182/blood-2002-06-1887.

34. Xiong J, Wang K, Yuan C, Xing R, Ni J, Hu G, et al. Luteolin protects mice from severe acute pancreatitis by exerting HO-1-mediated anti-inflammatory and antioxidant effects. Int J Mol Med 2017; 39: 113-125, doi: 10.3892/ijmm.2016. 2809.

35. Liu SH, Ma K, Xu XR, Xu B. A single dose of carbon monoxide intraperitoneal administration protects rat intestine from injury induced by lipopolysaccharide. Cell Stress Chaperones 2010; 15: 717-727, doi: 10.1007/s12192-0100183-0.

36. Bauer M, Huse K, Settmacher U, Claus RA. The heme oxygenase-carbon monoxide system: regulation and role in stress response and organ failure. Intensive Care Med 2008; 34: 640-648, doi: 10.1007/s00134-008-1010-2.

37. Zhao B, Fei J, Chen Y, Ying YL, Ma L, Song XQ, et al. Vitamin $C$ treatment attenuates hemorrhagic shock related multi-organ injuries through the induction of heme oxygenase-1. BMC Complement Altern Med 2014; 14: 442, doi: 10.1186/1472-6882-14-442.

38. Takahashi T, Kawano T, Eguchi S, Chi H, Iwata H, Yokoyama $M$. Effects of dexmedetomidine on insulin secretion from rat pancreatic beta cells. J Anesth 2015; 29: 396-402, doi: 10.1007/s00540-014-1943-2.

39. Jiang L, Qi Z, Li L, Shen J. Effect of dexmedetomidine hydrochloride on $\mathrm{H} 2 \mathrm{O} 2$-induced oxidative stress in alveolar macrophages. Zhong Nan Da Xue Xue Bao Yi Xue Bao 2013; 38: 1014-1019, doi: 10.3969/j.issn.1672-7347.2013. 10.007 . 